\title{
Designing a Web-based Telehealth System for Elderly People: An Interview Study in New Zealand
}

\author{
Jaspaljeet Singh Dhillon, Czarina Ramos, Burkhard C. Wünsche, Christof Lutteroth \\ Department of Computer Science \\ University of Auckland \\ Private Bag 92019, Auckland, New Zealand \\ \{jran055, cram021\}@ aucklanduni.ac.nz, \{burkhard, lutteroth\}@cs.auckland.ac.nz
}

\begin{abstract}
Designing healthcare systems for the elderly is a challenging endeavour. Telehealth systems are gaining popularity among elderly users, but such applications are mostly doctor-centric and are predominantly used for managing and/or treating diseases instead of preventing them. They do little to motivate patients to change their lifestyle and proactively manage their health. To address these shortcomings, we propose a web-based telehealth system, which uses Web 2.0 technologies in order to add social support and user defined content. In this study, we determine the functional and interface requirements of the system by conducting open individual semi-structured interviews with eight elderly people of age range 60 to 87. A paper prototype and three interfaces of existing Web 2.0 health applications were used to determine the optimum user interface design of the system. A qualitative content analysis was used to evaluate the responses. Overall, the respondents were positive about the idea of using the web to manage their healthcare from home and made several suggestions such as including applications to manage their diet, physiotherapy exercises to improve their health conditions and simple network games to reduce loneliness.
\end{abstract}

\section{Introduction}

Designing health systems for the elderly is challenging. Elderly users have special usability requirements due to possible functional impairment and inexperience in the use of computers. However, they need more support to manage their healthcare independently as they are known to be disproportionately more affected by chronic diseases, physical and mental challenges and loneliness. System designers need to focus on the users' capabilities and their specific requirements when developing healthcare systems.
Most teleheath solutions for elderly people are not webbased, but make use of standalone applications with specialized hardware. Generally, telehealth systems enable health care providers to deliver remote care and monitoring services to patients in their home. They are usually purchased by healthcare providers, and hence their design reflects the needs of the clinical user and of the purchasing entity. Patients are mainly an information source of health parameters. Telehealth systems are intended to lower costs and make effective use of existing healthcare resources. However, telehealth systems are generally focused on treating diseases instead of preventing them [7]. The applications do little to motivate patients to change their lifestyles and proactively manage their health. Many existing telehealth systems also suffer from high initial costs, cannot be extended by third parties, and require extra costs to add new functionalities [11]. They also do not address the social and psychological needs of the patient.

The emergence of Web 2.0 makes it possible to develop sophisticated health applications for health consumers. Web 2.0 health applications and services are rapidly gaining attention from patients and professionals, as they have the potential to extend traditional healthcare delivery models, empower patient self-care and provide social support. For instance, PatientsLikeMe (www.patientslikeme.com) is a popular website with more than 58,000 registered patients as of March 2010 [8], which provides access to valuable medical information aggregated from a large number of patients experiencing similar diseases. Apart from sharing health data and visualising patient data using charts, users are able to track various health parameters (e.g. weight, mood, etc.) that can be printed as a comprehensive report for doctors. Other consumer oriented web-based health support applications include Inspire, DailyBurn, DailyStrength, SugarStats and MyFitnessPal. Most of these systems are available for free, and target younger and middle aged users who are comfortable with computers. Many of the systems have 
crowded user interfaces with a plethora of functionalities, which makes it hard for the elderly to use them effectively. Further discussion about current Web 2.0 health applications can be found in $[6,8]$.

Recent research indicates that a large percentage of older generations, i.e. in the USA $78 \%$ of the 50 to 64 age group and $42 \%$ of the $65+$ age group, is using the web now [2]. Searching for health-related information is the third most popular online activity with the senior age group, after email and online search in general [10]. These data suggest that a web-based telehealth system for elderly users is of high interest in principle, however, the shortcomings of existing Web 2.0 healthcare applications need to be overcome.

Based on the limitations mentioned above, we have created a design for a web-based, patient-centric telehealth system called Healthcare4Life. It is ubiquitous, extendable by third parties, includes social aspects, and encourages cognitive engagement. In contrast to previous work, we propose an open structure with a middleware-like functionality. The framework emphasises the need for social support and psychological factors influencing usage and compliance. To keep overheads low, the framework supports the usage of common consumer level HCI devices such as webcam, keyboard, iPhone, and Wii remote controller with the system. We have developed the theoretical framework and a paper prototype. Currently, we are working on the first software prototype. More details of the Healthcare4Life framework can be found in [11].

To evaluate our design, we used the paper prototype and conducted systematic interviews with a group of elderly. The interviews had the following two objectives: 1) to determine the most important functional requirements, and 2) to find an optimal user interface. The functional requirements were determined by gaining an insight into the lifestyle of elderly people in terms of computer comfortability, healthcare management and socialisation. We have used the paper prototype and screenshots of existing Web 2.0 health applications to gain a good understanding of the interface requirements for Healthcare4Life. In this paper, we present the results of the interviews, which describe the functional requirements and perceptions of elderly people towards web-based health applications.

Several studies have been conducted in the past to identify better ways to design web interfaces for the elderly [5, 3], and organizations have published guidelines for designing web pages for seniors [1, 4]. However, most of the studies focus on user interface requirements in general, and do not discuss the specific requirements of web-based health applications. Our study sheds some light on these requirements in particular, and hence is potentially useful for all developers of such systems.

Section 2 presents the methodology used in conducting the study. Section 3 describes the interview results, and Section 4 discusses them. Section 5 concludes the paper.

\section{Methodology}

The study was designed as a qualitative inquiry focusing on elderly peoples' perceptions and requirements towards web-based health systems. Semi-structured interviews were conducted with each participant individually in respondents' homes between March and April 2011. Each interview lasted 45 to 60 minutes and participants were free to develop the conversation within each topic area. All interviews were tape recorded and anonymised. Notes were taken when the participant was elaborating the answer for each question. Impressions and gestures were carefully noted.

This semi-structured interview method was preferable over other interview methods because of its flexibility, i.e. allowing us to bring up new questions during the interview as a result of what the interviewee said. At the same time, the interviewee is free to share anything related to the conversation. Prior to the interviews, we prepared a framework of themes to be explored, including computer comfortability and usage, managing healthcare, and loneliness and socialisation. Response to these themes provide insight into elderly users' lifestyles, which helps to formulate the functional requirements of our system.

The interviews were made in an environment which was well known to the participants, and after each interview there was time for reflection. All the questions were asked verbally, with the exception of the oldest participant (age 87) who had a hearing impairment. For him, the questions were written down on paper.

For the second part of the interview, the paper prototype of Healthcare4Life and three screenshots of current Web 2.0 health applications were shown to the participants. First, an overview of how the web-based telehealth system will work was provided. Upon obtaining feedback on the paper prototype, the screenshots of the three health applications were described briefly. The homepages of these applications were printed in colour on A3 size paper. The purpose of the screenshots was to identify aspects users like and dislike about the interfaces, which helps towards creating an optimal user interface for our system. Three applications were selected because they address the general health of users and represent three different interface styles: MyFitnessPal (www.myfitnesspal.com), DailyBurn (www.dailyburn.com) and DailyStrength (www.dailystrength.org).

\subsection{Participants}

Eight elderly people ( 4 males and 4 females), who have basic experience in using computers were interviewed. The 
participant's mean age was 72 (range 60 to 87 ) years. Five participants were living in their own homes with families, and 3 were living in a retirement home. 4 participants were Indian, 3 were Caucasian and one was Asian.

\subsection{Data Analysis}

The interviews were analysed using qualitative content analysis [9]. The text was read and listened to first to acquire a first impression of the content. Thereafter, all the text was used in the analysis and divided into meaning-units corresponding to the specific themes. The frequency of a particular statement or similar statements was counted, and comments with the highest frequency were identified as important. Illustrative quotes were also highlighted.

\section{Results}

\subsection{Computer Comfortability and Usage}

Six out of eight participants reported using a computer almost daily. The oldest participant (age 87) commented that he used to be an active user but does not use the computer anymore as he wants to live a quiet life. Most of them are using a computer for email and Skype to keep in touch with their family and friends living overseas. One participant does not have an email account because she dislikes to type ("The computer is like my play partner. I use the computer to enjoy myself but I do not like typing”). To use Skype or any application which requires a username and password, she asks for help from her family members to log her on. The majority of the participants face problems in remembering their passwords, and they get annoyed with pop-ups. Other problems include websites with cluttered interfaces or small fonts, which causes them to close the site immediately.

\subsection{Managing Healthcare}

Six out of eight participants reported using the web to search for information related to their health. However, none trusted the information they found on the web completely, and hence discussed the findings with their physicians. Most of the time, the information found is regarded as less important or just added knowledge unless the source is reliable, such as an article provided by health professionals. The participants often searched for more information about any new prescription made by their physician (e.g. in order to learn about side effects).

No participant reported using a Web 2.0 health application, and only one played computer games. Four participants had heard about exercise games such as Wii Fit, but nobody used them.
All participants monitored their health by visiting their physician, with half of them doing visits at least every 3 months, and the rest only when not feeling well or prompted by their doctor. One participant visited the physiotherapist every week due to a recent leg injury. Six out of eight participants cannot drive anymore and are depending on someone (a family member or the retirement home's driver) to bring them to see the doctor.

None of the participants was using telehealth applications to monitor their health. All of them depend on their physician to monitor their vital signs. The majority of them (5 participants) do regular walking to maintain their health. A few of them commented that they like to walk every day, but at times they are unable to do so due to the weather conditions. Other ways participants manage their health include: maintaining healthy food habits, being vegetarian, having a positive mindset, practising yoga or tai chi, playing indoor bowling, and going shopping.

Six participants agreed that family support is crucial for managing health. One participant commented that: "Without help from family, we can't sustain".

\subsection{Loneliness and Socialisation}

Only two out of eight participants shared that they feel lonely at times. One of the participant who lives by himself in the retirement home expressed: "My wife died 4 years back. I miss my wife very much so I feel lonely at times ever since then".

The computer was an important tool for socialising and staying in touch with friends. Skype was most popular (6 participants), with email coming second. One participant added that: "It is convenient to socialise by using the computer because we can't drive". Other ways to maintain social contacts included home visits, phoning and playing indoor bowling with other elderly people.

Five out of eight participants did not have a Facebook account. Most of them felt that Facebook was made for younger people and does not have sufficient users in their age group. Other reasons included privacy (i.e. sharing personal information with others), lack of user friendliness, and taking up too much time.

From the three participants using Facebook, one reported that her children created the page for her, and that she mostly visits the Facebook when she receives an email stating someone has commented on her page. The other two participants (who were husband and wife) explained that they have a shared account. They visit Facebook almost daily. They both like Facebook because of its "simplicity". One of them added that: "Even if a friend hasn't been in touch with us for a week, we can still see their activities and they can see ours". 


\subsection{Interfaces Requirements}

Among the 3 web interfaces tested in the user study, DailyBurn (see Figure 1) was liked most by the participants. Almost all of them (7 out of 8) like the colourful icons on the left. DailyBurn uses a good contrast of colours for most of the items presented on the interface. The colours attracted their attention immediately. One participant expressed that the colours are inviting ("Come read me"). Almost all of them prefer the use of icons with text rather than just text. Five participants got excited looking at the weight chart presented on the interface, and none of them was aware of such an application already being available online. Only one participant felt the interface was presented with too many colours.

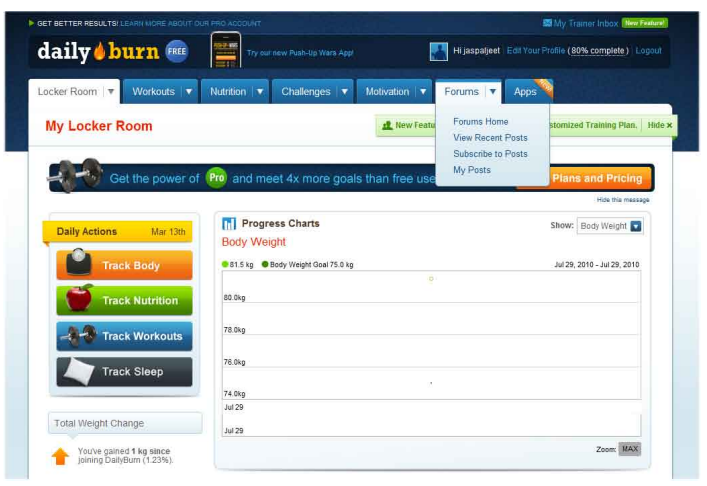

Figure 1. Top part of the main DailyBurn webpage.

MyFitnessPal (see Figure 2) had the second most popular interface. The study participants liked the "clear" layout. Three respondents like the idea of having the "Whats on your mind?" text box, just like Facebook. One participant expressed that this is a good feature because it enables users to share ("It is good to share, why do you want to keep it to your own self, let it go to others, they might benefit on something you say"). However, the interface uses less colours, i.e. mostly different tones of blue. Some the respondents feel that the items on the screen need to be more distinguishable, as one of the respondent expressed: "Use different color for different purpose". He also commented that: "It takes a bit time to identify which button is for what purpose". The page title, "News Feed - All Updates", could be misleading to the elderly. The oldest participant of the study thought it was containing some important news, i.e. like news on the television.

DailyStrength (see Figure 3) was the least popular interface in the study. The majority (6 respondents) felt it was too crowded, i.e. it contained too much information. One participant said: "It's tiring" and another participant
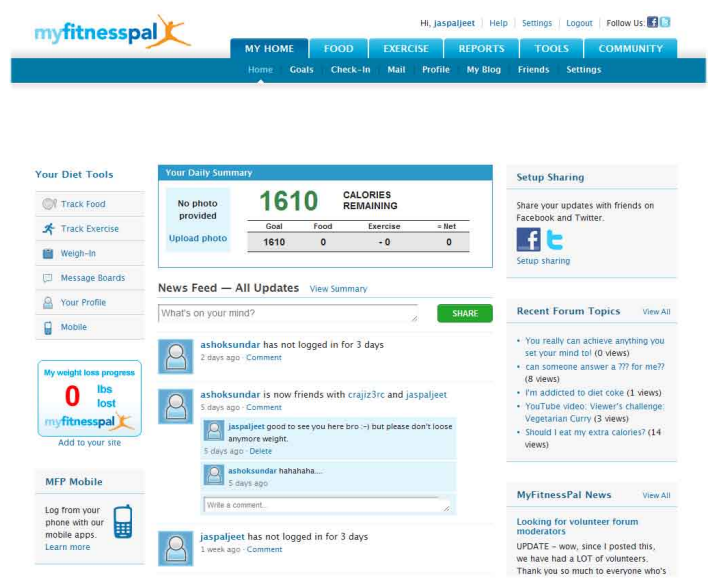

Figure 2. Top part of the main MyFitnessPal webpage.

suggested that: "The information should be straight to the point, e.g. my calories is this, my weight is this." There were too many links on the left. Also, the font size is too small for elderly. One of the participants liked the idea of having a chat box.

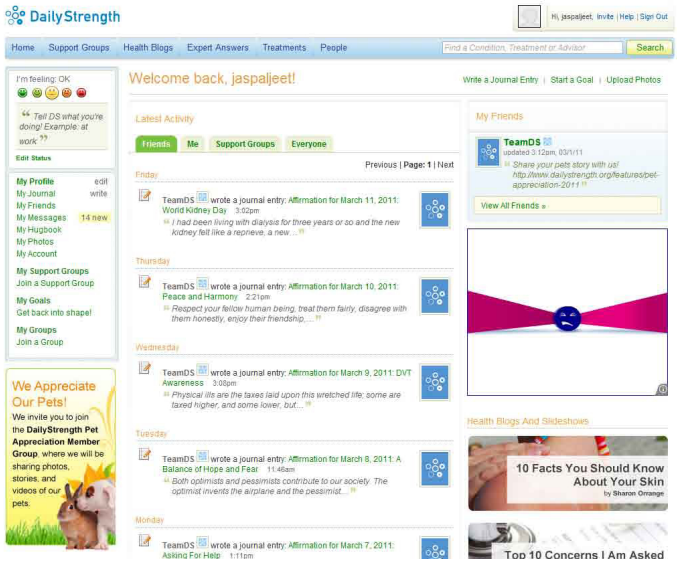

Figure 3. Top part of the main DailyStrength webpage.

The majority of participants preferred a menu bar at the top, rather than the left side, and light blue or green backgrounds. One of the participant expressed that: "Green is appealing for the eyes especially if we use it for long time. Another respondent expressed that: "Green is a suitable color for health systems, as it means hope and life".

We then showed the participants a paper prototype of our telehealth application, Healthcare4Life, which will consists of applications for monitoring, education, socialising, and rehabilitation (e.g. exercise games). In the prototype, users 
are expected to rate an application or write a review after using it. Most of the participants liked this idea, but one of the participants suggested that the rating should be made optional by clearly stating on the screen that it is not compulsory to rate the application. The participant felt that only health professionals (such as doctors) are able to rate health applications.

All participants generally liked the use of icons, but two participants found that not all the icons were suitable. One participant found that the plus sign in the icon for an "add applications" function looks like an icon for medicine or a red cross. Another participant thought the cartoon-like icon for the user profile page would be related to children. This indicates that elderly people may have different associations for some of the icons that have become commonplace on the web. Some participants were very concerned about privacy. One user suggested to mention clearly on the user profile page that all information entered there is strictly confidential. He mentioned that most people do not have the time to read the lengthy terms and conditions to find out about privacy. He suggested a lock icon to express that all data entered on a page are treated as confidential.

\subsection{Functional Requirements}

Almost all of the respondents expressed that the system should be user-friendly. The user should only be required to deal with one thing at a time. One participant commented that she will need assistance to use the system for the first time.

Half of them would like to make video calls to doctors from their homes, especially because they are not able to drive anymore. However, one of them was concerned about the cost of such a feature and some indicated that they prefer to see the doctor personally, since they want to monitor their health status (i.e. pulse rate, blood pressure, etc.).

Some of the respondents requested a reminder feature for doctor appointments and taking medication. The reminder should be synchronised with their mobile phone since they will not use their computer regularly.

Many respondents suggested that it will be very useful to have their health records transferred from the medical centres to the system. They want their health records to be accessible to them especially when they go overseas to meet their family members and friends. Note, however, that such a feature might conflict with regulatory requirements.

Several users suggested adding an application for diet management. One of the participants suggested: "The system should inform what your diet should be and how much you should eat for a particular health problem". Another participant recommended that the system should suggest health activities for specific health condition, illness or state of health. The participant added that we need to be careful with the choice of words used in the system ("The system should not use the term "illness", because people do not like to be called ill").

One elderly inquired whether the system will enable her to compare her health with her friends. She added that: "It will motivate if we can see if we are doing better than our peers". Other suggested features were applications to track health (e.g. weight, glucose and cholesterol), physiotherapy exercise for body pains (e.g. shoulder, back) and games (e.g. Majhong, Scrabble).

\section{Discussion}

Results from the interview show that most elderly are using the computer to communicate with their friends or to search for information related to their health, but none of the interviewed participants used web-based systems for monitoring or managing their health. Most users are not aware of existing Web 2.0 health applications and problems exist with interfaces and combining different types of functionalities. A web-based telehealth system should offer and integrate a large number of functionalities and present them with a simple, unified, easy-to-use interface.

Most of the participants prefer visualisation using graphs, which helps them to understand about their health easily, i.e. they can easily perceive changes in health parameters. They feel that it gives them satisfaction if, for example, the graph shows their weight has reduced, but will make them feel bad if their weight has increased. Care has to be taken to manage patient expectations, and provide encouragement and explanations.

Since most elderly agree that family support is crucial for managing their health, we strive to include members of users' families, social circles and support groups. For instance, an email alert about the activities done by the elderly can be sent to a family member or support person (nurse), who can encourage the user to do necessary tasks such as exercises or vital sign monitoring, and find out the reasons for not doing them.

The majority of the participants like the idea of managing their health using the computer, e.g. doing exercises via the computer to improve or maintain their health. By having such an application, they will be able to perform some exercises indoor without being constrained by weather conditions. However, one participant pointed out that there should be confirmation from the physician or health authority that a particular exercise in the system is suitable for a particular disease. Half of the respondents feel that it is important to keep track of all the activities they do to manage their health. One of the participant added that: "It will be very useful to have a record of what we have done and tell me what more I have to do more. We can share that information with doctors as normally we just mention we are 
doing exercise but we can't tell exactly how much we did". Although most of participants do not have interest in playing computer games, they are keen on playing simple health games (e.g. memory game) with their family members and friends over the Internet. One of the participants who lives alone commented: "I like the idea of playing over network because I do not have company here".

It was worth noticing the cultural differences between the participants. The Indian and Asian participants were living in their own homes with their children whereas the Caucasian participants had children but they choose to live independently in the retirement homes. The Asian participant and two Indian respondents shared that they are living with their own children, as it is their tradition that the children will take care of their parents when they grow old. Therefore, the lifestyles of elderly people are tied with their family values and traditions.

It is very important to design the pages in a linear way in order to guide users and simplify interactions. The interface needs to be attractive, clear and simple to use for the elderly. Our study suggests that elderly users prefer visuals over text. They like buttons or icons more than hyperlinks. If possible, every button (or icon) in the system should be presented with a suitable image and text. Every aspect of the system should be user-friendly and easy. Since remembering passwords is a major problem for elderly, better solutions must be found, e.g. by using graphical passwords (as suggested by some of the participants).

Interview results indicate that social support through Facebook-like features is useful, as long as an online community of like-minded users exist. Social interactions can be used to reduce loneliness, motivate each other and share experiences. One participant stated: "Talking is necessary to know other persons' disease, so that the person feels that I am not alone". Care must be taken that users choose professional advice for critical decisions, e.g. involving usage of medication.

One important result is that even existing computer users are less likely using computers with increasing age. Three older participants (range age 77 to 87 ) made it very clear that they support the idea of managing health via the webbased telehealth system but they are not sure if they will use the system. They are very comfortable with their existing practices of managing their health. In a way, they feel managing health using the computer is a burden.

\section{Conclusion}

Involving potential users of a system in its design is useful, especially when designing health systems for the elderly. Even though our study is based upon a small sample size, it provides a good insight into respondents' lifestyles, which helps to determine their expectations towards current web technologies to support their healthcare. The interview results support our hypothesis that the Internet is a suitable platform to deliver telehealth applications. Elderly users suggested several applications such as diet control, suitable exercises for different health problems, and simple network games to play with their families. They generally prefer a user interface layout which is clean, iconic and colorful, and uses a horizontal menu at the top, which makes it easy to identify and choose key functionalities. Our study also indicates that users reduce computer usage with increasing age and they are less likely to change their current practice of depending on their doctor for monitoring and managing their health. Web-based telehealth systems offer an opportunity to balance a reduction in healthcare costs with the needs of a rapidly aging society. More work is needed to create a user-friendly platform offering key services in one place and providing a suitable online community.

\section{References}

[1] Making Your Web Site Senior Friendly. Technical report, National Institute on Aging and the National Library of Medicine, 2006.

[2] Demographics of Internet Users. Technical report, Pew Research Center, 2010.

[3] D. Anson. The elder interface: Changing the computer experience by accommodating the sensory and motor changes of normal aging. The Elder Interface - Validation, 2006.

[4] D. Chisnell and J. G. Redish. Designing Web Sites for Older Adults: Expert Review of Usability for Older Adults at 50 Web Sites. AARP, 2005.

[5] G. Demiris, S. M. Finkelstein, and S. M. Speedie. Considerations for the design of a Web-based clinical monitoring and educational system for elderly patients. Journal of the American Medical Informatics Association : JAMIA, 8(5):46872, 2001

[6] J. S. Dhillon, B. C. Wünsche, and C. Lutteroth. Leveraging web 2.0 and consumer devices for improving elderlies' health. In Australasian Workshop on Health Informatics and Knowledge Management- Volume 120, HIKM '11, 2011.

[7] E. Dishman. Inventing wellness systems for aging in place. Computer, 37(5):34-41, May 2004.

[8] M. C. Domingo. Managing healthcare through social networks. Computer, 43(7):20-25, 2010.

[9] U. H. Graneheim and B. Lundman. Qualitative content analysis in nursing research: concepts, procedures and measures to achieve trustworthiness. Nurse education today, 24(2):105-12, Feb. 2004

[10] S. Jones and S. Fox. Generations Online in 2009. Pew Internet \& American Life Project, 2009.

[11] J. Singh, B. C. Wünsche, and C. Lutteroth. Framework for Healthcare4Life: a ubiquitous patient-centric telehealth system. In Proceedings of the 11th International Conference of the NZ Chapter of the ACM Special Interest Group on Human-Computer Interaction, CHINZ '10. ACM, 2010. 